\title{
Effect of shooting experience on executive function: Differences between experts and novices
}

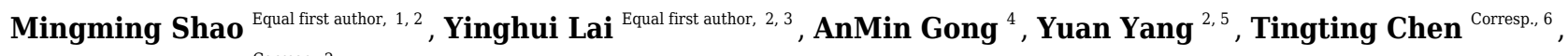 \\ Changhao Jiang ${ }^{\text {Corresp. } 2}$ \\ ${ }^{1}$ Faculty of Social and Public Administration, Guangdong Baiyun University, Guangzhou, China \\ 2 Beijing Key Laboratory of Physical Fitness Evaluation and Technical Analysis, Capital University of Physical Education and Sports, Beijing, China \\ 3 School of Education Science, Hunan Institute of Science and Technology, Yueyang, China \\ 4 School of Information Engineering, Engineering University of Armed Police Force, Xi'an, China \\ ${ }^{5}$ College of P.E. and Sports, Beijing Normal University, Beijing, China \\ ${ }^{6}$ School of Education, Beijing Dance Academy, Beijing, China \\ Corresponding Authors: Tingting Chen, Changhao Jiang \\ Email address: ctt1226@163.com, jiangchanghao@cupes.edu.cn
}

Background: Executive function (EF) plays an important role in controlling human actions. Shooting is a closed motor skill, characterized by high anti-interference ability and high mental intensity. However, the beneficial effects of closed exercises such as shooting on EF remain unclear.

Methods: We utilized an expert-novice paradigm and the Flanker task to examine the EF of shooting athletes. Participants were assigned into the novice group, expert group, or control group, based on the level of training and competition experience. Reaction time (RT) and accuracy of performance across the three groups were compared.

Results: For the simple task, the control group showed a longer RT than the novice group, for all three inter-stimulus interval (ISI) conditions. Significant differences between the control and the expert groups were observed only at 300-ms and 400-ms ISIs. For the complex task, the control group exhibited a higher RT than the novice and expert groups at the 300- and 400-ms ISIs.

Conclusions: The self-control during performing closed motor skills in the environment determines that shooters have proficient anti-interference ability. This ability is uncorrelated with task type, but most likely is affected by reserved task response time. 


\title{
Effect of shooting experience on executive function: Differences between experts and novices
}

\author{
Mingming Shao ${ }^{1,2 \S}$, YingHui Lai ${ }^{2,3 \S}$, AnMin Gong ${ }^{4}$, Yuan Yang ${ }^{2,5}$, Tingting Chen ${ }^{5}$, \\ ChanghaoJiang ${ }^{2}$ \\ ${ }^{1}$ Faculty of Social and Public Administration, Guangdong Baiyun University, Guangzhou, China \\ ${ }^{2}$ Beijing Key Laboratory of Physical Fitness Evaluation and Technical Analysis, Capital \\ University of Physical Education and Sports, Beijing, China \\ ${ }^{3}$ School of Education Science, Hunan Institute of Science and Technology, Yueyang, China \\ ${ }^{4}$ School of Information Engineering, Engineering University of Armed Police Force, Xi' an, China \\ ${ }^{5}$ College of P.E. and Sports, Beijing Normal University, Beijing, China \\ ${ }^{6}$ School of Education, Beijing Dance Academy, Beijing, China \\ $\S$ These authors contributed equally to this work \\ * Corresponding Authors: \\ Changhao Jiang, Ph.D ${ }^{1}$
}

No.11 North Third Ring Road West, Haidian District, Beijing 100191, P.R. China

Email address: jiangchanghao@,cupes.edu.cn

Tingting Chen, Ph.D ${ }^{2}$

No.105 West 3th Ring North Road, Haidian District, Beijing, 100048, P.R. China

Email address: ctt1226@163.com 


\section{ABSTRACT}

Background: Executive function (EF) plays an important role in controlling human actions. Shooting is a closed motor skill, characterized by high anti-interference ability and high mental intensity. However, the beneficial effects of closed exercises such as shooting on EF remain unclear.

Methods: We utilized an expert-novice paradigm and the Flanker task to examine the EF of shooting athletes. Participants were assigned into the novice group, expert group, or control group, based on the level of training and competition experience. Reaction time (RT) and accuracy of performance across the three groups were compared.

Results: For the simple task, the control group showed a longer RT than the novice group, for all three inter-stimulus interval (ISI) conditions. Significant differences between the control and the expert groups were observed only at 300-ms and 400-ms ISIs. For the complex task, the control group exhibited a higher RT than the novice and expert groups at the 300- and 400-ms ISIs.

Conclusions: The self-control during performing closed motor skills in the environment determines that shooters have proficient anti-interference ability. This ability is uncorrelated with task type, but most likely is affected by reserved task response time.

\section{Keywords}

executive function, shooting experience, expert-novice paradigm 


\section{INTRODUCTION}

2 Executive function (EF) is an advanced cognitive function, through which the brain coordinates

3 all available resources and controls the processes required to accomplish the specific goals of 4 complex cognitive tasks (Perner \& Lang, 1999). EF includes the processes of inhibition, working 5 memory, cognitive flexibility, and planning. EF processes have different developmental 6 trajectories, and the intensive development of each process begins at different periods. Each component is expected to contribute uniquely to an individual's abilities to resolve peer conflict competently, through the inhibition of incompetent social responses (response inhibition), the maintenance of social goals (working memory), and attentional shifts between complex social rules and potential response options (cognitive flexibility). Numerous studies have shown that exercise can have significant positive impacts on EF.

Motor skills can be categorized into open and closed (Dai et al., 2013; Tsai \& Wang, 2015). Open motor skills, such as table tennis, basketball, and football refer to skills that can be altered by changes in the individual's external context and that require the individual to process external information and predict upcoming events (Di Russo et al., 2010). In a longitudinal study, Kida et al. (2005) used a go/no-go paradigm to demonstrate that 2 years of baseball practice improved EF in college students. In some cross-sectional studies, Nakamoto and Mori (2008) reported that the reaction time (RT) of basketball and baseball players, in simple RT and go/no-go RT tasks, were shorter than those of non-players. Furley and Memmert (2012) showed that high-level basketball players can better resist external stimuli interference, focus on the current task, and make reasonable tactical decisions than lower-level basketball players. These studies have shown that open motor skills can have positive impacts on EF.

EF allows athletes to resist strong internal tendencies and external temptations; control attention, behavior, thinking, and emotions; and focus on the present to make appropriate behavioral decisions (Diamond, 2013). Closed motor skills refers to skills that can be performed without reference by changes in external conditions, such as yoga, shooting, and swimming. And closed motor skills generally have fairly fixed patterns of action. Recent reviews have documented the beneficial effects of closed motor skills on EF (Bowden et al., 2011). A randomized, controlled trial revealed that an acute bout of Hatha yoga resulted in significant improvements in working memory and selective attention, based on the Digit Letter Substitution task (Telles et al., 2012). Maeshima (2017) showed that synchronized swimming has beneficial effects on cognitive function, particularly with regards to recent memory. Players focused on closed (or open) motor skills require the ability to quickly suppress their motor responses and make new decisions.

To ensure a normal level of performance, athletes who participate in shooting sports must be 
36 control") (Sattlecker et al., 2014; Ihalainen et al., 2015). To achieve desired results, athletes must 37 continuously monitor their behaviors to ensure that they meet the requirements of the sport. 38 However, mistakes or errors may be made during practice and competition and it is important to minimize their effects on the outcome. After an error occurs, the athlete should promptly and accurately identify the problem ("emotion control") and make appropriate adjustments to ensure future accurate performance ("behavior control"). For example, during a skeet shooting competition, the athlete must pay attention to the direction the appearing target and its trajectory, and be able to adjust his/her shooting posture to achieve a successful hit. Emotion control and behavioral control enable the athletes to make quick and effective decisions to overcome negative impacts caused by previous errors or poor performances.

Shooting is a closed motor skills, characterized by high anti-interference ability and high mental intensity. The shooting movement has a particularly high requirement for executive and restraining functions. In order to achieve the target performance during a competition, the athlete often adjusts his/her current behavior based on previous experience. Similar to the conflict adaptation effect (CAE) of Flanker task, this cognitive behavior refers to the phenomenon that an individual can better solve conflicts in the process of human cognitive control if he/she experiences similar conflicts later. Therefore, this study adopted the classic paradigm of the Flanker task and hoped to recognize the relationship between shooting practice and EF through this paradigm. Participants with three levels of shooting experience were recruited: college graduate students without shooting experience (control group), shooting athletes with limited training and competition experience (novice group), and shooting athletes who had participated in international competitions or been among the top players in national competitions (expert group). We hypothesized that the reaction time (RT) would not differ among the three groups for performance of consistency or simple tasks; but for performing inconsistency or complex tasks, the expert group would exhibit the highest accuracy and fastest RT, followed by the novice group and control group.

\section{METHODS}

\section{Participants}

63 Thirty-two (32) right-handed participants were recruited, based on their shooting experience. The control group included 12 graduate students (mean age $=23.50 \pm 0.34$ years, 9 males), who had 0 year shooting experience. The novice group consisted of 9 athletes (mean age $=17.89 \pm 1.15$ years, 6 males), who had on average $2.84 \pm 0.57$ training and competition experience. The expert group contained 11 athletes on the Chinese national Clay Pigeon shooting team (mean age $=29.36 \pm .71$ years, 9 males), who had participated in international competitions or were among the top achievers in past national competitions (see Table 1). On average, the athletes in the expert group 
70 had $18.13 \pm 2.48$ years of training experience at the time of the study. All subjects had normal or corrected-to-normal visual acuity and were right-handed. No individuals reported any history of neurological, cardiovascular or musculoskeletal disorders, nor did any report taking medications that might affect cognitive or neuromuscular functions. The Institutional Ethics Committee of the Capital University of Physical Education and Sports approved the study, and all participants signed an informed consent before joining the study. The participants were instructed to refrain from consuming caffeine and alcohol beverages and to get at least 8 hours of sleep the day before the experiment.

\section{8}

79

80

\section{Procedures}

The Flanker task was run by using the E-prime program on a laptop computer, with a 14-in display screen and a resolution matrix of $1024 \times 768$. The background color was black. The fixation point, "+" and the stimulus were white and were located at the center of the screen. The sizes of the fixation point and the stimuli were $0.6 \mathrm{~cm}$ and $29 \mathrm{~cm} \times 10 \mathrm{~cm}$, respectively. The target stimulus consisted of five arrowheads. Two categories of stimuli were established: consistent $(>>>>>,<$ $<<<<$ ) and inconsistent $(<<><<,>><>>$ ) (see Figure 1). The participants were asked to judge the orientation of the middle arrowhead and to ignore the arrowheads flanking the target. If the middle arrowhead was oriented to the left, the participant used their left index finger to press the F key. If the middle arrowhead was oriented to the right, the participant used the right index finger to press the $\mathrm{J}$ key. The participants was required to maintain the fingers on the response buttons ( $\mathrm{F}$ and $\mathrm{J}$ keys) during the entire Flanker task. The experiment was performed in a quiet and well-lit laboratory.

The experiment adopted a reversal design (ABAB design) and was divided into one training module and four experimental modules. During the experimental modules, the first and third modules included consistent or simple tasks, whereas the second and fourth modules had inconsistent or complex tasks. Each experimental module comprised 36 trials over $30 \mathrm{~s}$, and the four modules together comprised 144 trials over $120 \mathrm{~s}$. The tasks required each participant to judge orientation of the middle arrows as quickly and accurately as possible. A rest period of at least 30 $\mathrm{s}$ was provided between two experimental modules to prevent the influence of the previous experimental module on the following module. Each experimental module began with a 500-ms introduction period, with the fixation point located on the center of the screen. The target stimulus was then presented for $150 \mathrm{~ms}$, consistent or inconsistent. The interstimulus intervals (ISI) were $200 \mathrm{~ms}, 300 \mathrm{~ms}$, and $400 \mathrm{~ms}$. The participant was required to react as quickly as possible within the ISI. The RT was recorded as the time from the stimulus presentation to the time that the response button was pressed. Simple and complex stimuli with RTs less than 100 ms were excluded from the analysis as they were out of human RT range. 


\section{Statistical analysis}

106 The data were analyzed using a 3 (shooting experience: control group, novice group, expert group)

$107 \times 2$ (task type: consistent, inconsistent) $\times 3$ (ISI: $200 \mathrm{~ms}, 300 \mathrm{~ms}, 400 \mathrm{~ms}$ ) analysis of covariance

108 (ANCOVA), in which shooting experience was a between-group variable, task type, and ISI were

109 within-group variables, and age was a covariate. RT and accuracy were dependent variables. SPSS

11023.0 statistical software was used to perform all statistical analyses. Partial eta-squared $\left(\eta^{2}\right)$ was

111 reported, to provide an overall association index for the proportion of total variance accounted for

112 by any treatment effect. The value range for partial $\eta^{2}$ is $0.00-1.00$. According to Shieh (2015), a

113 larger partial $\eta^{2}$ value reflects a stronger association between factors and dependent measures for

114 empirical studies.

\section{RESULTS}

116 The effects of shooting experience on the cognitive inhibition of EF are summarized in Table 1.

117 Table 2 presents the RTs for the consistent and inconsistent tasks in the three groups, and Table 3

118 presents the accuracy results for the two tasks in the three groups.

\section{Reaction time}

120 The RT data were subjected to 3 (shooting experience groups) $\times 2$ (task types) $\times 3$ (ISI) 121 repeated measures ANCOVA (see Figure 2, Table 4). The results showed that the main effect of

122 shooting experience was significant $\left[F(2,28)=9.441, p<0.001, \eta^{2}=0.403\right]$ and that the 123 interaction between shooting experience and ISI was significant $\left[F(4,56)=8.28, p<0.001, \eta^{2}=\right.$ $1240.372]$. The interaction between shooting experience and task type was also significant $[F(2,56)$ $\left.125=3.552, p<0.05, \eta^{2}=0.113\right]$. No other main effects or interactions reached significance.

126 For consistent trials, the pairwise comparison of groups with ISI showed that for the 200-ms 127 ISI, a significant difference was observed between the novice and control groups $(p=0.028)$, with 128 the control group showing longer RTs than the novice group. Differences among other groups 129 were not significant. For the 300-ms ISI, significant differences were seen between the expert and 130 control groups $(p=0.015)$ and between the novice and control groups $(p=0.010)$; the difference 131 between the expert and novice groups was not significant, and the control group showed longer 132 RTs than the expert and novice groups. For the 400-ms ISI, the results were similar to those for 133 the 300-ms ISI condition.

134 For inconsistent trials, the pairwise comparison of groups with ISI showed that for the 200135 ms ISI, no significant differences among groups were observed. For the 300-ms ISI, significant 136 differences were observed between the expert and control groups $(p=0.041)$ and between the 137 novice and control groups $(p=0.037)$, with the control group showing longer RTs than the expert 
138 and novice groups; no significant RT differences were seen between the expert and novice groups.

139 For the 400-ms ISI, the results were similar to those for the 300-ms ISI condition.

\section{Accuracy}

141 A 3 (shooting experience groups) $\times 2$ (task types) $\times 3$ (ISI) repeated measures ANCOVA for 142 accuracy data revealed that only the main effect of task type was significant (Figure $3 ; F(1,26)=$ $\left.1436.364, p<0.05, \eta^{2}=0.197\right)$, suggesting that the accuracy of the Flanker effect was not affected by 144 both the shooting experience and ISI. No other main effects or interactions reached statistical 145 significance.

\section{DISCUSSION}

147 The results of behavioral experiments showed that for the simple task, both the novice and expert groups demonstrated faster RT than the control group for all three ISI conditions. For the complex task, the RT results were consistent with those observed for the simple task. However, these differences were not found in the accuracy measurement. Therefore, the improved behavioral responses of the novice and expert group compared with the control group were primarily due to shooting experience.

Our behavioral data do not fully support our research hypothesis. The accuracy recorded for all tasks is in line with our hypothesis. Previous open motor skills studies that examined the inhibitory control of athletes found that athletes, such as baseball and basketball players, responded faster or committed fewer errors compared to non-players (Nakamoto \& Mori, 2008). The performance of a simple task only requires a quick instinctive response without a significant cognitive process. Our results indicate the absence of individual differences among the three groups. However, in another study of disabled athletes, comparisons among open-sport and closedsport athletes and healthy non-athletes showed that, although the RT was slower in disabled participants, the accuracy was similar in all three groups (Di Russo et al., 2010). In addition, these studies provides support for the argument that long-term training improves EF (Cheng et al., 2017; Di Fronso et al., 2016; Kim, Lee, Kim, \& Woo, 2013). Chan et al. (2011) reported that high-fitness fencers showed superior inhibitory control relative to non-athletes, whereas low-fitness fencers did not display improved inhibitory control. In contrast, Chun-Hao et al. (2013) reported that open motor skill athletes (tennis players) had shorter stop-signal RT compared with swimmers and sedentary controls, whereas no difference was found between the closed motor skill athletes (swimmers) and sedentary controls.

Additionally, the RT results were contrary to our hypothesis but instead conform to the 
171 reported that during simple consistency tasks, the RT of novice and expert tennis players did not 172 differ (Etnier, Nowell, Landers, \& Sibley, 2006; Kovacs, 2007). The discrepancy between our

173 findings and those of previous studies may be due to the use of Clay Pigeon shooters in our study.

174 Clay Pigeon shooters must complete a series of actions, including gunning, aiming, firing, and

175 shooting, within a 0.4-0.6-second period. Therefore, Clay Pigeon shooting requires a faster

176 response among shooting athletes.

177 The results showed no significant difference between novice and expert groups for the simple 178 task, which is consistent with previous studies. Clay Pigeon shooting has the shortest theoretical 179 training period among all shooting sports. For example, the normal training period for men's pistol 180 slow shooting is 10 years, whereas the average training period for Clay Pigeon shooting is only 2 181 years. In other words, with 2 years of hard training, individuals can improve their Clay Pigeon 182 shooting performance to the professional level. The training experience of the shooters in the 183 novice group in this study was at least 2 years. This result also confirmed the research hypothesis. 184 For complex task, the RT of the expert group was longer than that of the control and novice groups. 185 This result demonstrates that training can improve EF and is consistent with the findings of 186 previous studies (Kida et al., 2005; Nakamoto \& Mori, 2008).

187 This study demonstrates improved inhibitory function in closed motor skill athletes. 188 According to previous research, open motor skills and closed motor skills have different effects 189 on EF. Therefore, investigators should perform further research to examine dual sports at the 190 longitudinal study.

\section{CONCLUSION}

192 The self-control during performing closed motor skills in the environment determines that shooters 193 have proficient anti-interference ability. This ability is not correlated with task type, but most likely 194 is affected by reserved task response time. In the short ISI, participants in the three groups are 195 impossible to quickly and accurately respond to the stimuli, which leads to a"floor effect" of 196 behavioral performance. In contrast, the longer ISI reserves an adequate reaction time.

\section{FUNDING}

198 This work was supported by the National Natural Science Foundation (NNSF) of China under 199 Grant No 31771244, Research Fund of Beijing Institute of Sports Science (2017) and Research 200 Fund of Beijing Institute of Sports Science (19YTA001), and Education work committee of the 201 Beijing committee of the communist party of China [grant number 2016000020124G094]; and 202 Beijing education committee [grant number SM201810029001]. 


\section{ACKNOWLEDGMENTS}

204 This work was an open manuscript to be reviewed Research Fund of the State Key Laboratory of 205 Cognitive Neuroscience and Learning to CJ. Thanks for the help of Beijing Shooting School and 206 China National Shooting Team Coach Xiao Wang, and Professor Guanghui Yue from Kessler 207 Rehabilitation Center of the United States. 


\section{REFERENCES}

209 Bowden, D., Gaudry, C., An, S. C., \& Gruzelier, J. (2011). A comparative randomised controlled

210

211

212

213

214

215

216

217

218

219

220

221

222

223

224

225

226

227

228

229

230

231

232

233

234

235

236

237

238

239

240

241

242 trial of the effects of brain wave vibration training, iyengar yoga, and mindfulness on mood, well-being, and salivary cortisol. Evidence-Based Complementray and Alternative Medicine, e24-e25.

Chan, J. S. Y., Wong, A. C. N., Liu, Y., Yu, J., \& Yan, J. H. (2011). Fencing expertise and physical fitness enhance action inhibition. Psychology of Sport and Exercise, 12(5), 509514.

Cheng, M. Y., Wang, K. P., Hung, C. L., Tu, Y. L., Huang, C. J., Koester, D., \& Hung, T. M. (2017). Higher power of sensorimotor rhythm is associated with better performance in skilled air-pistol shooters. Psychology of Sport and Exercise, 47-53.

Dai, C. T., Chang, Y. K., Huang, C. J., and Hung, T. M. (2013). Exercise mode and executive function in older adults: an ERP study of task-switching. Brain and Cognition, 83(2), 153 162.

Diamond, A. (2013). Executive functions. Annual Review of Psychology, 64(1), 135-168.

Etnier, J. L., Nowell, P. M., Landers, D. M., \& Sibley, B. A. (2006). A meta-regression to examine the relationship between aerobic fitness and cognitive performance. Brain Research Reviews, 52(1), 119-130.

Fronso, S. D., Robazza, C., Filho, E., Bortoli, L., Comani, S., \& Bertollo, M. (2016). Neural markers of performance states in an olympic athlete: An EEG case study in Air-Pistol shooting. Journal of Sports Science and Medicine, 15(2), 214-222.

Furley, P. A., \& Memmert, D. (2012). Working memory capacity as controlled attention in tactical decision making. Journal of Sport and Exercise Psychology, 34(3), 322-344.

Ihalainen, S., Kuitunen, S., Mononen, K., \& Linnamo, V. (2015). Determinants of elite-level air riflfle shooting performance: Air riflfle shooting performance. Scandinavian Journal of Medicine \& Science in Sports. 26.

Kida, N., Oda, S., \& Matsumura, M. (2005). Intensive baseball practice improves the Go/Nogo reaction time, but not the simple reaction time. Cognitive Brain Research, 22(2), 257-264.

Kim, W., Lee, G., Kim, J., \& Woo, M. (2013). A comparison of cortico-cortical communication during air-pistol shooting in elite disabled and non-disabled shooters. Personality and Individual Differences, 54(8), 946-950.

Kovacs, M. S. (2007). Tennis physiology: training the competitive athlete. Sports Medicine, 37(3), 189-198.

Nakamoto, H., \& Mori, S. (2008). Sport-specific decision-making in a Go/Nogo reaction task: Difference among nonathletes and baseball and basketball players. Perceptual and Motor 
243

244

245

246

247

248

249

250

251

252

253

254

255

256

257

Skills, 106(1), 163-170.

Russo, F. D., Bultrini, A., Brunelli, S., Delussu, A. S., Polidori, L., Taddei, F., \& Spinelli, D. (2010). Benefits of sports participation for executive function in disabled athletes. Journal of Neurotrauma, 27(12), 2309-2319.

Sattlecker, G., Buchecker, M., Müller, E., Lindinger, S.J. (2014). Postural balance and riflfle stability during standing shooting on an indoor gun range without physical stress in different groups of biathletes. International Journal of Sport Science and Coaching. 9 (1), 171-183.

Telles, S., Bhardwaj, A. K., Kumar, S., Kumar, N., \& Balkrishna, A. (2012). Performance in a substitution task and state anxiety following yoga in army recruits. Psychological Reports. $110(3), 963$.

Tsai, C. L., and Wang, W. L. (2015). Exercise-mode-related changes in task-switching performance in the elderly. Frontiers in Behavioral Neuroscience. 56.

Wang, C. H., Chang, C. C., Liang, Y. M., Shih, C. M., Chiu, W. S., Tseng, P., \& Juan, C. (2013). Open vs. closed skill sports and the modulation of inhibitory control. PLOS ONE, 8(2). 


\section{Figure 1}

An example of stimulus

The flanking arrows either all pointed in the same direction as the target arrow (e.g., " $<<<<<$ "), or they all pointed in the opposite direction (e.g., " $<<><<$ "). The trials on which the flanking arrows pointed in the same direction as the target arrow were the congruent trials $(A)$; the trials in which they pointed in the opposite direction were the incongruent trials (B). Subjects were to press " $F$ " button for a left facing central arrow and "J" button for a right facing central arrow.

\section{(A)}

(B)

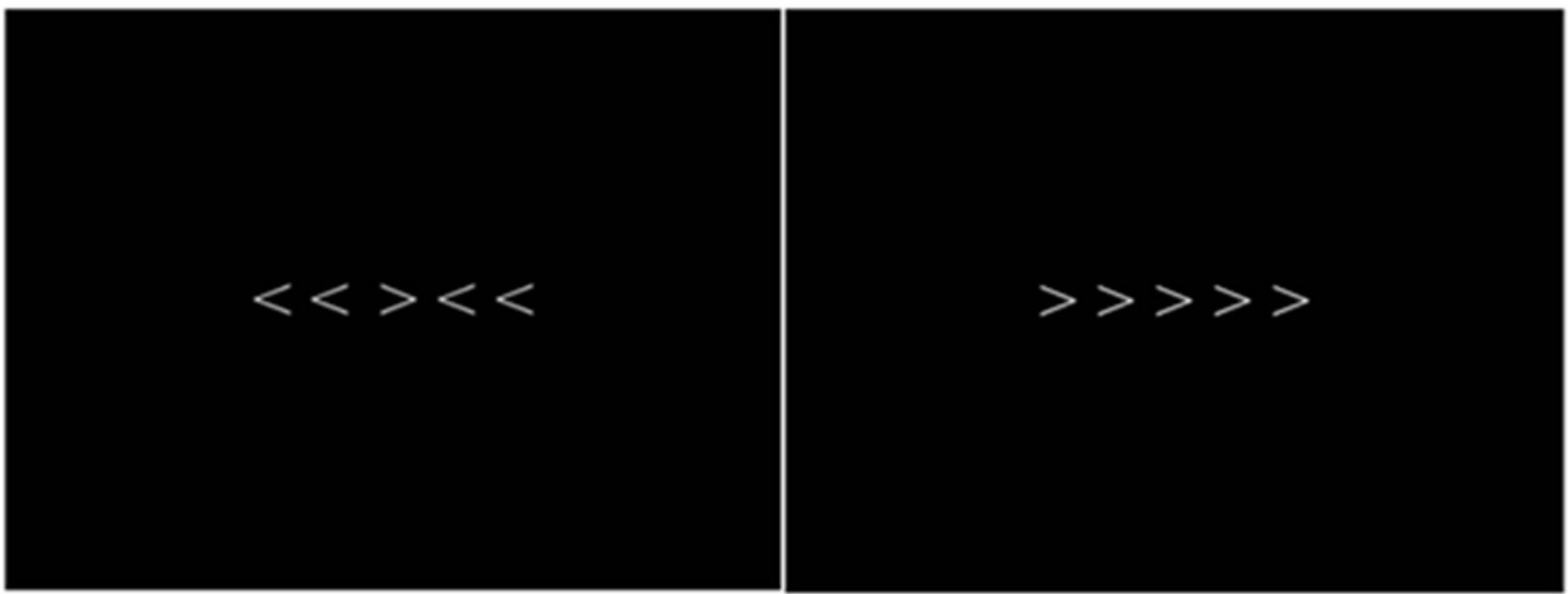


Figure 2

\section{Reaction Time (ms)}

Effects of the interaction between shooting experience and task type on RT by ISI 200 (A) $\square$ ISI 300 (B) and ISI 400 (C).

(A) ISI200

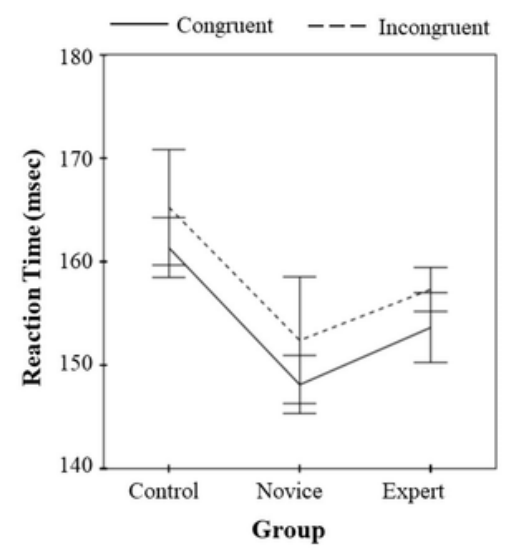

(B) ISI300

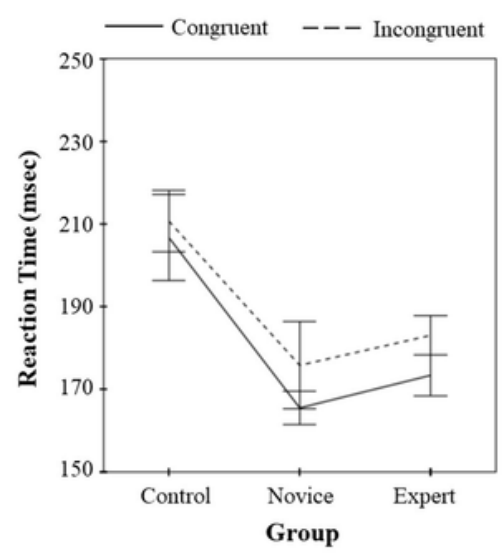

(C) ISI400

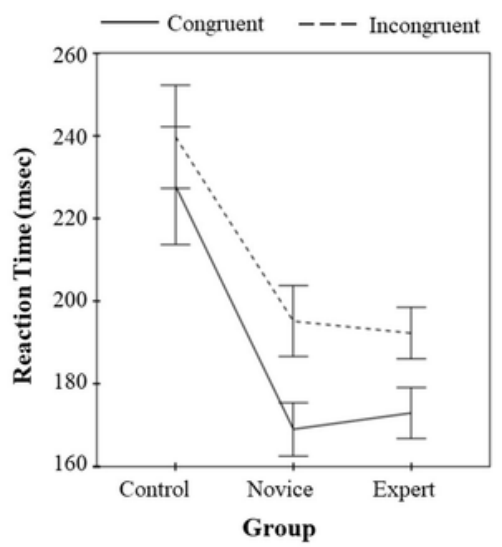




\section{Figure 3}

\section{Accuracy Rate}

Effects of the interaction between shooting experience and task type on ACC by ISI 200 (A) $\square$ ISI 300 (B) and ISI 400 (C).

(A) ISI200

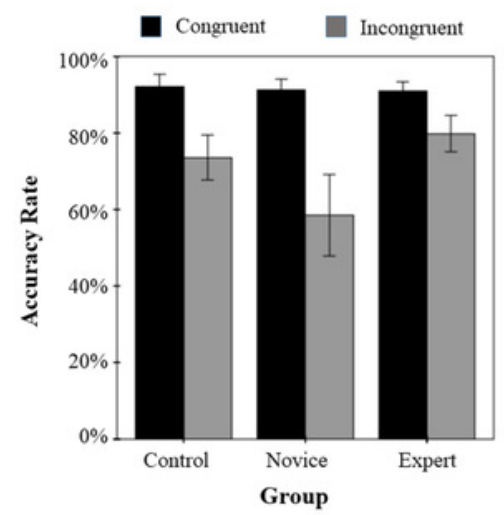

(B) ISI300

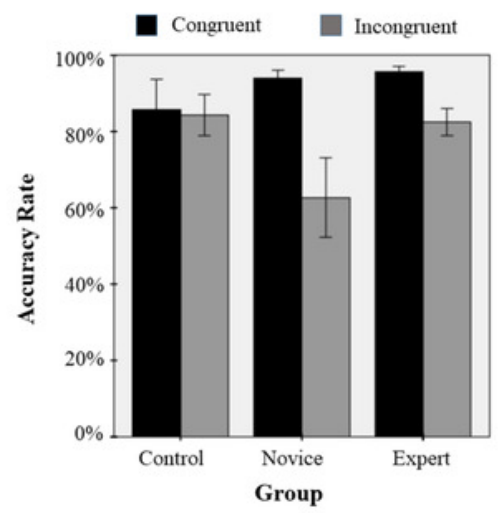

(C) ISI400

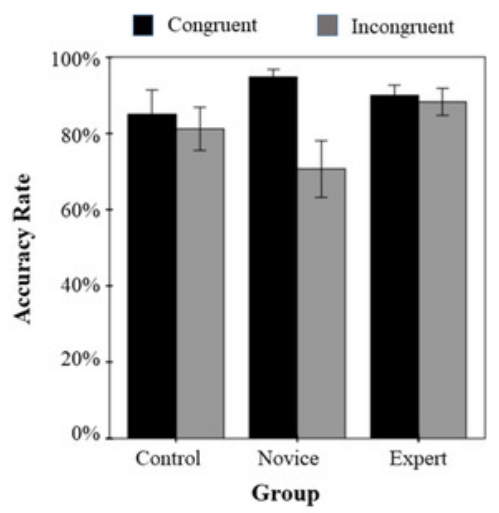




\section{Table $\mathbf{1}$ (on next page)}

Descriptive statistics for behavioral data

Basic information about participants, such as gender and age stratification 


\begin{tabular}{lcccc}
\multirow{2}{*}{ Group } & Age (years) & Shooting age (years) & \multicolumn{2}{c}{ Sex } \\
\hline control group & $23.50 \pm 0.34$ & 0 & 9 & Female \\
novice group & $17.89 \pm 1.15$ & $3.39 \pm 1.04$ & 6 & 3 \\
expert group & $29.36 \pm 2.71$ & $14.27 \pm 2.66$ & 9 & 2 \\
\hline
\end{tabular}


Table 2 (on next page)

Descriptive statistics for Reaction time(ms) data

Mean Reaction time (ms) with standard errors for each condition. 


\begin{tabular}{cccccccc}
\hline \multirow{2}{*}{$\begin{array}{c}\text { Group } \\
\text { Accuracy }\end{array}$} & \multicolumn{3}{c}{ Consistent Task } & & \multicolumn{3}{c}{ Inconsistent Task } \\
\cline { 2 - 3 } \cline { 7 - 8 } & $200 \mathrm{~ms}$ & $300 \mathrm{~ms}$ & $400 \mathrm{~ms}$ & & $200 \mathrm{~ms}$ & $300 \mathrm{~ms}$ & $400 \mathrm{~ms}$ \\
\hline Control Group & $0.91 \pm 0.12$ & $0.93 \pm 0.06$ & $0.94 \pm 0.08$ & & $0.74 \pm 0.19$ & $0.91 \pm 0.07$ & $0.85 \pm 0.18$ \\
Novice Group & $0.91 \pm 0.09$ & $0.94 \pm 0.06$ & $0.95 \pm 0.06$ & & $0.59 \pm 0.32$ & $0.63 \pm 0.31$ & $0.71 \pm 0.22$ \\
Expert Group & $0.91 \pm 0.08$ & $0.96 \pm 0.05$ & $0.90 \pm 0.09$ & & $0.80 \pm 0.16$ & $0.82 \pm 0.12$ & $0.88 \pm 0.12$ \\
Total & $0.91 \pm 0.09$ & $0.94 \pm 0.06$ & $0.93 \pm 0.08$ & & $0.71 \pm 0.24$ & $0.79 \pm 0.22$ & $0.82 \pm 0.19$ \\
\hline
\end{tabular}


Table 3(on next page)

Descriptive statistics for Accuracy data

Mean accuracy rate with standard errors for each condition. 


\begin{tabular}{cccccccc} 
Group & \multicolumn{3}{c}{ Consistent Task } & & \multicolumn{3}{c}{ Inconsistent Task } \\
\cline { 2 - 4 } \cline { 6 - 7 } RT $(\mathrm{ms})$ & $200 \mathrm{~ms}$ & $300 \mathrm{~ms}$ & $400 \mathrm{~ms}$ & & $200 \mathrm{~ms}$ & $300 \mathrm{~ms}$ & $400 \mathrm{~ms}$ \\
\hline Control & $161.34 \pm$ & $206.74 \pm$ & $227.92 \pm$ & & $165.26 \pm$ & $210.70 \pm$ & $239.78 \pm$ \\
Group & 10.03 & 36.18 & 49.46 & & 19.39 & 25.93 & 43.29 \\
Novice & $148.11 \pm$ & $165.50 \pm$ & $169.05 \pm$ & & $152.39 \pm$ & $175.82 \pm$ & $195.15 \pm$ \\
Group & 8.41 & 11.97 & 19.38 & & 18.33 & 31.65 & 25.68 \\
Expert & $153.63 \pm$ & $173.39 \pm$ & $172.97 \pm$ & & $157.32 \pm$ & $183.05 \pm$ & $192.28 \pm$ \\
Group & 11.21 & 16.37 & 20.45 & & 7.11 & 15.85 & 20.63 \\
Total & $154.97 \pm$ & $183.68 \pm$ & $192.47 \pm$ & & $158.91 \pm$ & $191.39 \pm$ & $210.90 \pm$ \\
& 11.16 & 30.45 & 43.36 & & 16.29 & 28.59 & 38.61 \\
\hline
\end{tabular}


Table 4 (on next page)

ANCOVA TEST(RT) 
1

\begin{tabular}{ccccc}
\hline & $\mathrm{df}$ & $\mathrm{F}$ & $p$ & $\eta^{2}$ \\
\hline age & 1 & 0.422 & 0.521 & 0.015 \\
group & 2 & 9.441 & 0.001 & 0.403 \\
ISI & 2 & 1.837 & 0.169 & 0.062 \\
ISI * age & 2 & 0.397 & 0.674 & 0.014 \\
ISI * group & 4 & 8.280 & 0.000 & 0.372 \\
task & 1 & 2.140 & 0.155 & 0.071 \\
task * age & 1 & 0.246 & 0.624 & 0.009 \\
task * group & 2 & 0.688 & 0.511 & 0.047 \\
ISI * task & 2 & 3.552 & 0.035 & 0.113 \\
ISI * task * age & 2 & 1.946 & 0.152 & 0.065 \\
ISI * task * group & 4 & 0.512 & 0.727 & 0.035 \\
\hline
\end{tabular}

